BULL. AUSTRAL. MATH. SOC.

VOL. $2(1970), 117-124$.

\title{
Structure of perfect rings
}

\section{Vlastimil Dlab}

In the present note, we offer a simple characterization of perfect rings in terms of their components and socle sequences, which is subsequently used to establish a one-to-one correspondence between perfect rings and certain finite additive categories. This correspondence is effected by means of a matrix representation, which describes the way in which perfect rings are built from local perfect rings.

The concept of a perfect ring was introduced by S. Eilenberg in [2]; later, in his paper [1], H. Bass characterized perfect rings in several ways. As our starting point, refer to Theorem $P(1)$ of [1] and call a ring $R$ (right) perfect if

(a) $R / \operatorname{Rad} R$ is artinian (i.e. completely reducible)

and

(b) $\operatorname{Rad} R$ is $T$-nilpotent in the sense that, given any sequence $\left\{\rho_{i}\right\}$ of elements of $\operatorname{Rad} R$, there exists an $n$ such that $\rho_{n} \rho_{n-1} \cdots \rho_{2} \rho_{1}=0$.

In what follows, $R$ denotes a ring with unity; by a module $M$ we always understand a (left unital) $R$-module. The symbol Rad $M$ stands for the intersection of all maximal submodules of $M$ if there are any; otherwise $\operatorname{Rad} M=M$. Dually, if $M$ has minimal submodules, Soc $M$ denotes their union; if $M$ has no minimal submodules, Soc $M=0$. In a ring $R$, define the (left transfinite) socle sequence

Received 3 November 1969. 


$$
0=S^{(0)} \subseteq S^{(1)} \subseteq \ldots \subseteq S^{(\alpha)} \subseteq \ldots \subseteq R
$$

of two-sided ideals $S^{(\alpha)}$ by

$$
S^{(\alpha)} / S^{(\alpha-1)}=\operatorname{Soc} R / S^{(\alpha-1)} \text { for all non-limit }
$$

and

$$
S^{(\alpha)}=\bigcup_{\beta<\alpha} S^{(\beta)} \text { for all limit ordinals } 1 \leq \alpha .
$$

If $R=S^{(\delta)}$ for a certain $\delta, R$ is said to have a socle sequence. It is easy to see that $R$ has a socle sequence if and only if Soc $M \neq 0$ for every $R$-module $M \neq 0$. This, in turn, is equivalent to the fact that every non-zero monogenic $R$-module possesses a minimal submodule, i.e. that, for every proper left ideal $L$ of $R$, the $R$-module $R / L$ contains a minimal submodule.

PROPOSITION 1. A ring $R$ has a socle sequence if and only if $R / \operatorname{Rad} R$ has a socle sequence and $\operatorname{Rad} R$ is T-nilpotent. Thus, in particular, if $R$ has a socle sequence, then $\operatorname{Rad} R$ is nil.

Proof. Using the argument of $H$. Bass [1] p. 470, the implication "if" follows easily. In order to prove the opposite assertion, consider a proper left ideal $L$ of $R$ and notice that $\operatorname{soc} R / L \neq 0$ if $\operatorname{Rad} R \subseteq L$. Also, $R / L$ has obviously a minimal submodule provided that $R / L \cap$ Rad $R$ has one. Hence, we may assume that $L \nsubseteq \operatorname{Rad} R$.

Suppose that $R / L$ has no minimal submodule. Then, we can construct a sequence $\left\{\rho_{i}\right\}$ of elements of $\operatorname{Rad} R$ in the following way: Take $\rho_{1} \in \operatorname{Rad} R \backslash L$ and assume that we have already chosen $\rho_{2}, \ldots, \rho_{n} \in \operatorname{Rad} R$ such that

$$
\alpha_{n}=\rho_{n} \rho_{n-1} \cdots \rho_{2} \rho_{1} \notin L \text {. }
$$

Thus $R \alpha_{n} \notin L ;$ moreover, we can show that also $(\operatorname{Rad} R) \alpha_{n} \notin L$. For, if $(\operatorname{Rad} R) \alpha_{n} \subseteq L$, then the non-zero submodule $R \alpha_{n}+L / L$ of $R / L$ which is isomorphic to $R \alpha_{n} / R \alpha_{n} \cap L$ would be a homomorphic image of $R \alpha_{n} /(\operatorname{Rad} R) \alpha_{n}$ and thus a homomorphic image of $R / \operatorname{Rad} R$. Therefore $R / L$ would possess 
a minimal submodule. Hence, we may choose $\rho_{n+1} \in \operatorname{Rad} R$ such that $\alpha_{n+1}=\rho_{n+1} \alpha_{n} \nmid L$. However, the existence of such a sequence $\left\{\rho_{i}\right\}$ contradicts the $T$-nilpotence of $\operatorname{Rad} R$. The proof is completed.

Notice that using Proposition $I$ we can deduce easily that $R$ is perfect if and only if (a) holds and $R$ has a socle sequence (cf. J.P. Jans [3]).

PROPOSITION 2. Let $R=L \oplus T$ with an indecomposable left ideat $L$ and let $R$ have a socle sequence. Then $L$ contains a unique left ideal $K$ of $R$ maximal in $L$. Thus, in particular, $R$ is an indecomposable ring which has a socle sequence if and only if $R$ is a local (i.e. possessing a unique maximal left ideal) perfect ming.

Proof. Let $\{\lambda, \tau\}$ be a complete set of orthogonal idempotents of $R$ corresponding to the decomposition $R=L \oplus T$, and $\{\bar{\lambda}, \bar{\tau}\}$ the respective set of idempotents of $R / \operatorname{Rad} R$. Obviously, $K=\operatorname{Rad} L q L$. Take a left ideal $S$ of $R, K \subset S \subseteq L$, such that $S / K$ is a minimal submodule of $L / K$. By definition of $K$, there is a left ideal $W$ of $R, K \subseteq W \subset L$, maximal in $L$ and such that $S \cap W=K$. Our Proposition will be proved if we show that $W=K$, i.e. that $S=L$. As sume that $W \neq K$. Then $R / \operatorname{Rad} R \cong L / K \oplus T / \operatorname{Rad} T=S / K \oplus W / K \oplus T / \operatorname{Rad} T$; moreover, $S / K$ contains an idempotent $\bar{\sigma}_{0}$. Consider the set consisting of the idempotents $\bar{\sigma}=\bar{\lambda} \bar{\sigma}, \bar{\omega}=\bar{\lambda}-\bar{\sigma}$ and $\bar{\tau}$; arguments of a routine nature yield that it is a complete set of orthogonal idempotents of $R / \operatorname{Red} R$. In view of Proposition 1, we can lift these idempotents modulo $\operatorname{Rad} R$ and we get

$$
R=R \sigma \oplus R \omega \oplus T,
$$

contradicting the indecomposability of $L$. The proof is completed.

THEOREM 1. A ring $R$ is (wight) perfect if and only if

(a*) $R=\bigoplus_{i=1}^{r} L_{i}$ with indecomposable (left) ideals $L_{i}, \quad 1 \leq i \leq r$, and

(b*) $R$ has a socle sequence. 
A ring $R$ satisfying $\left(a^{*}\right)$ and $\left(b^{*}\right)$ has the property that each component $L_{i}$ contains a unique left ideal $K_{i}$ of $R$ maximal in $L_{i}$,

$$
\begin{gathered}
\operatorname{Rad} R=\bigoplus_{i=1}^{r} K_{i}, \\
R / \operatorname{Rad} R \cong \bigoplus_{i=1}^{r} L_{i} / K_{i}
\end{gathered}
$$

and thus, the decomposition of $R$ in $\left(a^{*}\right)$ is unique up to an isomorphism and order of the components.

Proof. In view of our definition of a perfect ring, both $\left(a^{*}\right)$ and (b*) follow easily from Proposition $I$ and the fact that idempotents modulo Rad $R$ can be lifted.

Now, if $R$ satisfies $\left(a^{*}\right)$ and $\left(b^{*}\right)$, then in view of Proposition 2 each $L_{i}$ contains a unique maximal left ideal $K_{i}$,

$\operatorname{Rad} R=\bigoplus_{i=1}^{r} \operatorname{Rad} L_{i}=\bigoplus_{i=1}^{r} K_{i}$ and the uniqueness of the decomposition in (a*) follows from the uniqueness of the decomposition $\underset{i=1}{\oplus} L_{i} / K_{i}$ of $R / \operatorname{Rad} R$ combined with the fact that $L_{i} / K_{i} \simeq L_{j} / K_{j}$ for $1 \leq i, j \leq r$ implies $L_{i} \simeq L_{j}$. In particular, $R$ satisfies (a) and is therefore perfect.

PROPOSITION 3. Let $R=\underset{i=1}{\oplus} L_{i}$ be an indecomposable decomposition of a perfect ring $R$. Then, for each $i, 1 \leq i \leq r$, the endomorphism ring $\operatorname{End}_{R}\left(L_{i}\right)$ of $L_{i}$ is a local perfect ring.

Proof. Without loss of generality, take $i=1$ and denote by $K_{1}$ the unique left ideal of $R$ maximal in $L_{1}$. First, notice that

$$
\left\{\varphi \mid \varphi \in \operatorname{End}_{R}\left(L_{1}\right) \text { and } L_{1} \varphi \subseteq K_{1}\right\}
$$

is the unique (Ieft) maximal ideal of $E_{1}=\operatorname{End}_{R}\left(L_{1}\right)$ and hence $E_{1}$ is local.

In order to establish that $E_{1}$ satisfies $\left(b^{*}\right)$, let us take an 
arbitrary left ideal $A$ of $E_{1}$ and show that the $E_{1}$-module $E_{1} / A$ has a minimal $E_{1}$-submodule. To this end, consider the "standard" matrix representation $\Delta$ of the ring $R$ corresponding to our decomposition: $R$ is isomorphic to the ring $R \Delta$ of all $r \times r$ matrices $\left(x_{i j}\right)$ with $x_{i j} \in \operatorname{Hom}_{R}\left(L_{i}, L_{j}\right)$. Now, denote, for $2 \leq i \leq r$, by $N_{i l}$ the submodule of $\operatorname{Hom}_{R}\left(L_{i}, L_{1}\right)$ of all $a_{i l}$ such that

$$
x_{1 i} a_{i 1} \in A \text { for all } x_{1 i} \in \operatorname{Hom}_{R}\left(L_{1}, L_{i}\right) \text {. }
$$

And, observe that the set $X$ of all matrices $\left(a_{i j}\right) \in R \Delta$ such that $a_{i j}=0$ for $j \geq 2, a_{11} \in A$ and $a_{i 1} \in N_{i I}$ for $2 \leq i \leq r$, is a left ideal of $R \Delta$. Since $R$ is perfect, there is a left ideal $Y$ of $R \Delta$ such that $X \subseteq Y$ and $Y / X$ is a simple $R \Delta$-module. Obviously,

$$
A \nsubseteq\left\{x_{11} \mid\left(x_{i j}\right) \in Y\right\}=B \subseteq E_{1} \text {; }
$$

moreover, $B$ is a left ideal of $E_{1}$. And finally, $B / A$ is a simple $E_{1}$-module. For, otherwise there would be a left ideal $C$ of $E_{1}$ such that $A \subseteq C$ 必 $B$, and thus the left ideal $Z$ of $R \Delta$ of all matrices $\left(c_{i j}\right)$ such that $c_{i j}=0$ for $j \geq 2, c_{11} \in C$ and $c_{i l} \in N_{i 1}$ for $2 \leq i \leq r$, would satisfy $X \subseteq Z q Y$. The proof is completed.

Now, given a perfect ring $R$, consider its indecomposable decomposition $R=\bigoplus_{i=1}^{r} L_{i}$ and denote by $R \Phi$ the finite additive category whose objects are $R$-modules $L_{1}, L_{2}, \ldots, L_{r}$ and whose morphisms are all homomorphisms belonging to $\operatorname{Hom}_{R}\left(L_{i}, L_{j}\right), 1 \leq i, j \leq r$. Notice that the mapping $\Phi$ of the class of all perfect rings into the class of all finite additive categories is, in view of uniqueness of decomposition, well-defined. The image $R \Phi$ of every perfect ring $R$ is, moreover, $a$ category such that the endomorphism rings of its objects are local perfect rings. For the sake of brevity, let us call such finite additive categories perfect.

On the other hand, let $\mathcal{C}$ be a finite additive category; denote by 
$C_{1}, C_{2}, \ldots, C_{r}$ its objects. Define the ring $\mathcal{C} \Psi$ in the following way: $C \Psi$ is the ring of all $r \times r$ matrices $\left(x_{i j}\right)$ such that

$$
x_{i j} \in\left[C_{i}, C_{j}\right] \text { for all } 1 \leq i, j \leq r \text {, }
$$

with respect to matrix addition and multiplication.

PROPOSITION 4. If $C$ is a perfect category, then $C \Psi$ is a perfect ring.

Proof. For each $i, 1 \leq i \leq r$, denote by $w_{i}$ the unique maximal (left) ideal of the ring $\left[C_{i}, C_{i}\right]$ and by $L_{i} \subseteq C \Psi$ the subset of all matrices $\left(x_{i j}\right)$ such that

$$
x_{i j}=0 \text { for } j \neq i \text {, }
$$

which is obviously a left ideal of $C \Psi$. One can verify readily that the subset $K_{i} \subseteq L_{i}$ of those matrices $\left(x_{i j}\right)$ which satisfy

$$
\left[c_{i}, c_{k}\right] \times x_{k i} \subseteq w_{i} \text { for all } k, 1 \leq k \leq r
$$

is a unique left ideal of $C \Psi$ maximal in $L_{i}$. Hence $L_{i}$ are indecomposable and $C \Psi$ has property $\left(a^{*}\right)$.

In order to verify $\left(b^{*}\right)$, it is sufficient to show that, for every $i, 1 \leq i \leq r$, and every left ideal $X$ of $C \Psi$ contained properly in $L_{i}$, there exists a left ideal $Y, X \subset Y \subseteq L_{i}$ such that $Y / X$ is a simple $C \Psi$-module. The latter is trivial for $r=1$. Thus, assume that $r>1$ and, without loss of generality, present a proof for $i=1$. The left ideal $X \nsubseteq L_{1}$ consists evidently of all matrices $\left(x_{k j}\right) \in L_{1}$ such that $x_{11}$ belongs to a certain left ideal $X_{11}$ of $\left[C_{1}, C_{1}\right]$ and for each $k, 2 \leq k \leq r, x_{k I}$ belongs to a certain submodule $x_{k \perp}$ of $\left[c_{k}, C_{1}\right]$. Since $\left[c_{p}, c_{p}\right]$ is perfect, the $\left[c_{r}, c_{p}\right]$-module $\left[c_{r}, c_{r}\right] / X_{r l}$ has a simple submodule $y_{r l} / X_{r l}$. Denote by $x^{(r)}$ the left ideal of $C \Psi$ generated by the set of all matrices $\left(x_{k_{j}}\right) \in L_{1}$ with $x_{k 1} \in X_{k 1}$ for $1 \leq k \leq r-1$ and $x_{r l} \in y_{r l}$. Obviously, $X q_{\neq} X^{(r)} \subseteq L_{1}$. 
Furthermore, writing

$$
z_{k 1}^{(r)}=X_{k l}+\left[c_{k}, c_{r}\right] \times y_{r l}, I \leq k \leq r,
$$

we can see easily that $X^{(r)}$ consists of all matrices $\left(x_{k j}\right) \in L_{1}$ such that $x_{k I} \in z_{k I}^{(r)}$.

Now, if $z_{k 1}^{(r)}=X_{k 1}$ for all $k \leq r-1$, then $y=X^{(r)}$ satisfies the property that $Y / X$ is a simple $C \Psi$-module, and the proof is completed. Otherwise, denote by $x$ the greatest index $\leq r-1$ such that

$$
X_{s 1} \mathrm{Z}_{\varepsilon l}^{(r)},
$$

and by $Y_{s l}$ the $\left[C_{s}, C_{s}\right]$-submodule of $z_{s l}^{(r)}$ containing $X_{s l}$ such that $y_{s l} / X_{s l}$ is simple; such a submodule exists because the ring $\left[C_{s}, C_{s}\right]$

is perfect. Furthermore, let $x^{(s)}$ be the left ideal of $C \Psi$ generated by the set of all matrices $\left(x_{k l}\right) \in L_{1}$ with $x_{k I} \in X_{k l}$ for $1 \leq k \leq r$, $k \neq s$ and $x_{s I} \in Y_{s I}$. Obviously,

$$
X \subseteq X^{(s)} \subseteq X^{(r)} \subseteq L_{1}
$$

Again, write

$$
z_{k 1}^{(s)}=X_{k l}+\left[C_{k}, C_{s}\right] \times y_{s l} \text { for } 1 \leq t \leq s \text {, }
$$

and repeat the above argument. After a finite number of steps, we reach a lèft ideal $X^{(q)}$ of $C \Psi$ such that

$$
X \subseteq X^{(q)} \subseteq \cdots \subseteq X^{(s)} \subseteq X^{(r)} \subseteq L_{1}
$$

and such that $q=1$ or the corresponding

$$
z_{k I}^{(q)}=x_{k I} \text { for all } k \leq q-1 \text {. }
$$

In either case, $Y=X^{(q)}$ has the required property. The proof is completed. 
Now, we can formulate

THEOREM 2. There is a one-to-one correspondence between the non-isomorphic perfect rings and non-isomorphic perfect categories. This correspondence is effected by a matrix representation which describes the way in which perfect rings are built from local perfect rings.

Proof. Given a perfect ring $R$, the matrix ring $R \Phi \Psi$ is the "standard" matrix representation of $R$ and is thus isomorphic to $R$. Also, giten a perfect category $C$ and expressing $C \Psi=\underset{i=1}{\oplus} L_{i}$ as the direct sum of the column vectors $L_{i}$, we check easily that $C \Psi \Phi$ is isomorphic to the category $C$. The theorem follows.

\section{References}

[1] Hyman Bass, "Finistic dimension and a homological generalization of semi-primary rings", Trans. Amer. Math. Soc. 95 (1960), 466-488.

[2] Samuel Eilenberg, "Homological dimension and syzygies", Ann. of Math. $64(1956), 328-336$.

[3] J.P. Jans, "Some aspects of torsion", Pacific J. Math. 15 (1965), 1249-1259.

Carleton University,

Ittawa, Canada. 